\title{
Domestic Mammals as Reservoirs for Leishmania donovani on the Indian Subcontinent: Possibility and Consequences on Elimination
}

\author{
Anurag Kushwaha ${ }^{1}$, Breanna Scorza ${ }^{2}$, Om Prakash Singh ${ }^{1}$, Edgar Rowton ${ }^{3}$, Philip \\ Lawyer $^{3}$, Shyam Sundar ${ }^{1}$, and Christine Petersen ${ }^{2}$ \\ ${ }^{1}$ Banaras Hindu University Institute of Medical Sciences \\ ${ }^{2}$ University of Iowa \\ ${ }^{3}$ Walter Reed Army Institute of Research
}

December 31, 2020

\begin{abstract}
Leishmania donovani is the causative agent of historically anthroponotic visceral leishmaniasis (VL) on the Indian subcontinent (ISC). L. donovani is transmitted by the sand fly species Phlebotomus argentipes. Our collaborative group and others have shown that sand flies trapped outside in endemic villages have fed on cattle and dogs in addition to people. Domestic animals are reservoirs for L. donovani complex spp., particularly L. infantum, in other endemic areas. Multiple studies using quantitative PCR or serological detection methods have demonstrated that goats, cattle, rats and dogs were diagnostically positive for $L$. donovani infection or exposure in eastern Africa, Bangladesh, Nepal and India. There is a limited understanding of the extent to which $L$. donovani infection of domestic animals drives transmission to other animals or humans on the ISC. Evidence from other vector-borne disease elimination strategies indicated that emerging infections in domestic species hindered eradication. The predominant lesson learned from these other situations is that non-human reservoirs must be identified, controlled and/or prevented. Massive efforts are underway for VL elimination on the Indian subcontinent. Despite these herculean efforts, residual VL incidence persists. The specter of an animal reservoir complicating elimination efforts haunts the final push toward full VL control. Better understanding of $L$. donovani transmission on the Indian subcontinent and rigorous consideration of how non-human reservoirs alter VL ecology are critical to sustain elimination goals.
\end{abstract}

Title: Domestic Mammals as Reservoirs for Leishmania donovani on the Indian Subcontinent: Possibility and Consequences on Elimination

\section{Running title: Reservoirs of Leishmania donovani on Indian Subcontinent}

Anurag Kumar Kushwaha ${ }^{1}$, Breanna M. Scorza ${ }^{2}$, Om Prakash Singh ${ }^{3}$, Edgar Rowton ${ }^{4}$, Phillip Lawyer ${ }^{5}$, Shyam Sundar ${ }^{1 \#}$, Christine A. Petersen ${ }^{2,6 \#}$

${ }^{1}$ Department of Medicine, Institute of Medical Sciences, Banaras Hindu University, Varanasi, India. ${ }^{2}$ Department of Epidemiology, College of Public Health, University of Iowa, Iowa City, Iowa, USA. ${ }^{3}$ Department of Biochemistry, Institute of Science, Banaras Hindu University, Varanasi, India. ${ }^{4}$ Division of Entomology, Walter Reed Army Institute of Research, Silver Spring, Maryland, USA. ${ }^{5}$ Laboratory of Parasitic Diseases, National Institute of Allergy and Infectious Diseases, National Institute of Health, Bethesda, MD, USA. ${ }^{6}$ Center for Emerging Infectious Diseases, University of Iowa, Coralville, Iowa, USA.

\# corresponding authors:

Dr. Christine A. Petersen 
Department of Epidemiology, College of Public Health, University of Iowa,

Iowa City, Iowa 52242, USA. Center for Emerging Infectious Diseases, University of Iowa, Research Park, Coralville, Iowa 52241, USA. Email:

christine-petersen@uiowa.edu

\section{Prof. Shyam Sundar}

Department of Medicine, Institute of Medical Sciences,Banaras Hindu University

Varanasi-221 005, India. Phone No. +91 5422369632

Email: drshyamsundar53@gmail.com

Keywords: India, Psychodidae, visceral leishmaniasis, zoonoses

\section{Summary}

Leishmania donovani is the causative agent of historically anthroponotic visceral leishmaniasis (VL) on the Indian subcontinent (ISC). L. donovani is transmitted by the sand fly speciesPhlebotomus argentipes . Our collaborative group and others have shown that sand flies trapped outside in endemic villages have fed on cattle and dogs in addition to people. Domestic animals are reservoirs for L. donovani complex spp., particularly $L$. infantum, in other endemic areas. Multiple studies using quantitative PCR or serological detection methods have demonstrated that goats, cattle, rats and dogs were diagnostically positive for L. donovani infection or exposure in eastern Africa, Bangladesh, Nepal and India. There is a limited understanding of the extent to which $L$. donovaniinfection of domestic animals drives transmission to other animals or humans on the ISC. Evidence from other vector-borne disease elimination strategies indicated that emerging infections in domestic species hindered eradication. The predominant lesson learned from these other situations is that non-human reservoirs must be identified, controlled and/or prevented. Massive efforts are underway for VL elimination on the Indian subcontinent. Despite these herculean efforts, residual VL incidence persists. The specter of an animal reservoir complicating elimination efforts haunts the final push toward full VL control. Better understanding of $L$. donovani transmission on the Indian subcontinent and rigorous consideration of how non-human reservoirs alter VL ecology are critical to sustain elimination goals.

\section{Introduction}

Leishmaniases are a spectrum of sand fly-borne infectious diseases of humans spanning from localized cutaneous lesions to systemic disease. The causative agents of Leishmaniases are more than 20 species of the protozoan parasite genus Leishmania (Trypanosomatida :Trypanosomatidae ) (Burza et al., 2018, Espinosa et al., 2018).Leishmania spp. are often characterized as "Old World"; found in Eurasia, Africa and the Mediterranean basin, or "New World"; found in the Americas, based on their geographical distribution (McMahon-Pratt and Alexander, 2004, Reithinger et al., 2007). Old World Visceral Leishmaniasis (VL) is caused by $L$. donovani and $L$. infantum . New World VL is primarily caused by $L$. infantum(Marcili et al., 2014, Shaw, 2006, Monge-Maillo and López-Vélez, 2013). The epidemiology of leishmaniasis is based on ecological interactions between human and reservoir systems, parasite and sand fly species, regional characteristics of endemic areas, present and past susceptibility of human and animal populations and parasite and human behavior (Ready, 2008, Oryan and Akbari, 2016, Patz et al., 2000, Ready, 2013, Lukeš et al., 2007). The Leishmania life cycle is directly associated with ecological variables in rural or peri-domestic environments that shelter sand fly niches and reservoir hosts in conjunction with human living areas (Daszak et al., 2001). Leishmaniases are found across tropical, equatorial areas, with warm and rainy climates abetting reproduction of Phlebotomines (Fischer et al., 2011). Human activities such as migration, deforestation, unorganized urbanization or shifts in infection vulnerability caused by immunosuppression and malnutrition are reflected in significant increases in human susceptibility to leishmaniasis (Alvar et al., 2008, Desjeux, 2004, Thakur, 2000). 


\section{Anthroponotic spread of Leishmania and elimination strategy of VL on the ISC.}

The World Health Organization listed leishmaniasis in category I, Emerging or Uncontrolled Diseases. Although leishmaniasis is endemic in over 98 countries and territories, eco-epidemiological hotspots for visceral leishmaniasis (VL) occur in East Africa, the Indian sub-continent (ISC), and Brazil. More than 50\% of global VL incidence has been reported in India, Nepal and Bangladesh (Burza et al., 2018, WHO, 2020). VL is caused by $L$. donovani complex spp. protozoan parasites and manifest epidemiologically by two types of etiologies(Figure 1) : Anthroponotic visceral leishmaniasis (AVL), where parasites are transmitted by sand fly bites from human to human and occurs mainly on the ISC and in East Africa; and zoonotic visceral leishmaniasis (ZVL), which is transmitted from infected animals to humans, with domestic dogs as the primary reservoir host and occurring in Mediterranean countries, the Middle East, Asia, and South America(Figure 2) (Desjeux, 2004, Esch and Petersen, 2013). Despite these geographic separations, parasites within the $L$. donovanicomplex have a high level of genetic similarity, estimated to be greater than $99 \%$ (Franssen et al., 2020). This indicates that there are not likely to be DNA-encoded reasons for host-preference differences between L. donovani and L. infantum. Instead, theLeishmania life cycle is directly impacted by ecological variables in rural or peridomicile environments that shelter sand fly niches and reservoir hosts in proximity to humans. Leishmania donovani infection on the ISC has traditionally been recognized as an anthroponotic disease with a continuous cycle of transmission in and around human dwelling inhabited by vector sand flies.(Stauch et al., 2011, Malaviya et al., 2011). Active VL patients are thought to be the primary reservoir for AVL on the ISC (Figure 3), based on the clustering of cases around households with past VL history (Barnett et al., 2005, Bern et al., 2005, Das et al., 2016).

National programs to eliminate VL were initiated by the governments of India, Bangladesh, and Nepal in 2005 as a major public health problem on the ISC. The goal of these programs was to reduce the incidence of VL at the sub-district or district level to less than $1 \mathrm{VL}$ case per 10,000 population per annum by the 2015 (WHO, 2005). A key strategy of the VL elimination agenda has been systematic Indoor Residual Spraying (IRS), targeting sand flies feeding on people within their homes, asPhlebotomus argentipes is the only recognized $L$. donovaniinsect vector on the ISC. This serves as one of the prongs in a three-pronged approach; in addition to early active case identification, and comprehensive treatment of clinical cases to eliminate VL (Huda et al., 2012, Mondal et al., 2009). Although IRS campaigns have made considerable progress in reducing VL in India, Nepal, and Bangladesh, they fell short of the 2015 elimination target and are not on track to meet the extended deadline set for 2020 (Programme, 2017, Rijal et al., 2019). It is possible that these shortcomings are influenced by zoonotic sources of transmission, which to date have not been considered in intervention strategies on the ISC.

\section{Challenges of elimination and possible infection sources on the ISC.}

An assumption behind the elimination campaign is that only symptomatic human VL and post kala-azar dermal leishmaniasis (PKDL) cases are infectious to P. argentipes on the ISC (Figure 3). However, this hypothesis has not been completely evaluated, and is challenged by the possible existence of latent, asymptomatic human reservoirs of the parasite and potential animal reservoirs (Stauch et al., 2011, Molina et al., 2020, Singh et al., 2014). Asymptomatic VL cases are much more common than clinical cases, as shown in highly endemic villages in India and Nepal (Ostyn et al., 2011, Gidwani et al., 2011). Because of this high frequency, the role of asymptomatic individuals in VL transmission should be assessed to effectively identify human reservoirs in South Asia (Bern et al., 2010). Additionally, co-infection with HIV is a significant threat to VL management. Molina et al . used xenodiagnosis as a tool for evaluating VL infection in HIV+ patients and concluded that early-stage asymptomatic VL patients with HIV infection were infectious to sand flies (Molina et al., 2020). Further, transmission studies suggest PKDL patients are a significant parasite source for sand flies, particularly in immunocompromised people (Kamhawi and Serafim, 2017). PKDL is a form of VL sequelae where papular or macular dermal lesions occur that can be extensively parasitized and/or arise in response to immune reactions against persistent dermal parasites in treated VL patients (Zijlstra et al., 2017, Mukhopadhyay et al., 2014). As another reservoir of infection, PKDL patients represent a major health risk for disease resurgence in that skin parasite load is a strong predictor of positive xenodiagnosis 
(Mondal et al., 2019, Molina et al., 2017) (Figure 3).

Risk factors for VL on the ISC include migration, proximities of households to animal shelters, and crowded conditions (Custodio et al., 2012, Bern et al., 2010, Ranjan et al., 2005). Livestock density in India is regarded as a threat variable for increasing human VL incidence (Barnett et al., 2005). The phenomenon of multiple frustrated feeding attempts seen by Leishmania -infected sand flies means a vector may feed on several nearby hosts including livestock (Bern et al., 2005, Courtenay et al., 2017). Indeed, the presence of cattle has been shown to attract or sustain higher sand fly densities, thus indirectly contributing to human infection through human-sand fly exposure. The prevalence of outdoor P. argentipes combined with the inclination of ISC residents to sleep outdoors during peak sand fly season correlate with an increased risk of VL (Perry et al., 2013, Picado et al., 2012).

In Chad, epizootics in domestic animals aggravated Guinea worm eradication efforts and indicated that dogs may have been a source of worms acquired by humans (Thiele et al., 2018, Eberhard et al., 2014). The presence of an animal reservoir raised the question of whether these animals were the culprits hampering eradication efforts (Galán-Puchades, 2017, Callaway, 2016). While it is widely known that L. donovaniinfection is transmitted between people, however, there has been little attention paid to the possibility of infections of domestic animals on the ISC despite genetically similar Leishmania species having a known zoonotic transmission ecology, (Esteva et al., 2017, Quinnell and Courtenay, 2009). Mathematical models are an important tool to understand disease transmission dynamics and assess elimination viability. Nevertheless, most models are limited by data gaps of critical biological parameters such as duration of immunity, infectiousness of different disease stages, comorbidities leading to recrudescent disease and transmission etc. (Esteva et al., 2017, Coffeng et al., 2019). A recent xenodiagnosis study on asymptomatic VL subjects in the Mediterranean basin (Molina et al., 2020) and India (Singh et al., 2020b) indicated serologically positive asymptomatic people were not the cause for ongoing transmission (Figure 3), suggesting that there could be an alternative non-human host sustaining transmission. These models conclude that xenodiagnosis experiments and more clinical data are necessary to predict the role of asymptomatic infection, PKDL, and domestic animals as infection reservoirs on the ISC (Tiwary et al., 2017, Le Rutte et al., 2016, Singh et al., 2020a).

\section{Role for domestic animals in the current ecology of disease.}

Reservoir hosts are an integral part of the Leishmania parasite life cycle. The pathogen - vector - reservoir relationship is complex and reveals regional and temporal variation (Raymond et al., 2003). Several studies suggest that wild, domestic and synanthropic mammals including rock hyraxes, rats, foxes, pigs, cats can be infected and dogs have been identified as reservoir host for Leishmania (Akhoundi et al., 2016, Palatnikde-Sousa and Day, 2011, Rohousova et al., 2015, Dereure et al., 2000). A reservoir species is characterized as one or more epidemiologically bound populations where a pathogen can be permanently established as an infection and from which an infecting organism is transmitted to the target population (Haydon et al., 2002). Identification of mammalian Leishmania reservoirs has occurred by demonstrating that the mammal could harbor parasites in its blood (parasitemia) through culture and more recently through PCR/qPCR, while in many cases, their role as a reservoir has not been confirmed(Table 1). Mammalian reservoirs are required for transmission of zoonotic and rural/sylvatic infections to sustain the life cycle of multiple Leishmaniaspecies (Esteva et al., 2017). VL is assumed to have only an anthroponotic transmission cycle on the ISC since there is still no direct evidence of non-human mammalian hosts infectious to the sand fly vector (Stauch et al., 2011, Singh et al., 2010). Phlebotomus argentipes, the principle vector of VL in India, aggregates in and around animal housing, which offers a favorable, stable micro-climate near a blood meal and may alter disease dynamics by altering vector/host contact rates (Keeling, 1999). Several research studies indicate that IRS has altered $P$. argentipes feeding behavior from endophilic to exophilic and sand flies are most likely to feed on outdoor livestock in areas where IRS occurs (Cameron et al., 2016). Alternative blood sources for exophilic P. argentipes may include cattle, goats, dogs, or rodents (Poche et al., 2018) (Figure 1). Importantly, PCR evidence of $L$. donovani parasitemia has been found in cattle, buffalo, and goats (Bhattarai et al., 2010).

The previous conclusion that there is no animal reservoirs for L. donovani on the ISC is brought into 
question by observations of anti-Leishmania specific antibodies and Leishmaniaquantitative PCR positivity detected from domestic animal blood(Table 1). Dogs have been incriminated as a bridge between the sylvatic cycle of Leishmania and humans. Investigators believe dogs were responsible for the zoonotic cycle ofLeishmania (Courtenay et al., 2002b, Courtenay et al., 2002a). In Brazil dogs are the primary reservoir host for L. infantum (Esch et al., 2012), a visceralizing Leishmania species genetically very similar to $L$. donovani (Courtenay et al., 1994, Blackwell et al., 2009, Akhavan et al., 2010). Canine Leishmania infection in VL-endemic areas of Bangladesh and Sudan. Another recent study in India found L. donovani DNA in the blood of local dogs, goats and cows, indicating that these are potential animal reservoirs of VL(Table 1) . Rodents and dogs are both classical reservoir species for other L. donovani complex spp. Other less traditional reservoirs caused outbreaks of $L$. infantum within certain foci (Molina et al., 2012, Ruiz-Fons et al., 2013). Importantly, natural zoonotic infections with $L$. donovani have been identified in dogs, other domestic animals and rodents in other areas outside the ISC(Figure 2). A study of the emergence of VL in Middle Eastern and Mediterranean countries indicated that high prevalence of infected dogs, in the presence of a competent vector species, led to the onset of human disease (Hamarsheh et al., 2012, Nasereddin et al., 2005). Besides being the primary reservoir for zoonotic VL in many endemic regions, the presence of infected dogs was a critical risk factor predisposing humans to infection in these locations (Gavgani et al., 2002). The epidemiology of vector-mediated transmission from dogs and other non-traditional reservoir species on the ISC requires closer inspection to evaluate their potential role. The totality of $L$. donovani reservoirs needs to be more widely understood to protect $L$. donovanielimination goals (Cameron et al., 2016).

\section{Conclusion}

To effectively control VL on the ISC, a more global understanding of its epidemiology and regional transmission dynamics is needed. Management of potential domestic and sylvatic zoonoses would involve implementation of a more complex elimination strategy than exists under the current framework, which considers humans as the only reservoir of ISC $L$. donovani . Previously only considered anthroponotic, $L$. donovanihas recently been shown to have non-human mammalian hosts by molecular and serological studies in many countries. As domestic mammals are a common documented source of sand fly blood meals, the contributions of livestock as parasite hosts or reservoirs for $L$. donovani remains an open question for additional investigation. Xenodiagnosis is the most practical way to assess transmissibility of parasites from a potential host to sand flies, and can confirm the role of these populations in the emerging non-human ecology of $L$. donovani . These host-transmission insights are critical to reinforce models forecasting VL outbreaks on the ISC and worldwide.

This article should serve as a catalyst for epidemiologists, entomologists, veterinarians, physicians, parasitologists and immunologists to come together and evaluate critical interactions between sand flies, mammalian hosts and $L$. donovani leading to transmission on the ISC. The main question is not how domestic animals become infected with $L$. donovani but rather: to what extent does $L$. donovani circulate in domestic species after being fed upon by infected $P$. argentipes ? Instead of performing costly active surveillance to look for the lack of clinical VL cases, we should instead consider how can we best monitor transmission (or lack thereof) within the whole potential $L$. donovani ecosystem. Infection outcome is determined by parasite virulence as well as by host genetic predisposition and immune/health status of the host. Infection can be controlled by removing exposure to the parasite through insecticides or reducing the parasite burden through chemo and/or immunotherapy (Baneth et al., 2008). Surveillance is complicated by the long incubation period of $L$. donovani in mammals, leading to periods of latency, when infection can be difficult to detect to prevent transmission. Interdisciplinary collaboration and broadly based ecological surveillance of all potential P. argentipes blood meal sources and xenodiagnosis studies will be required for some time to rule out zoonotic contributions and achieve L. donovani transmission eradication goals on the Indian Subcontinent.

\section{Acknowledgments}

This work was supported by the Extramural Program of the National Institute of Allergy and Infectious Diseases, National Institutes of Health (Tropical Medicine Research Center grant U19AI074321). The funders had no role in design, decision to publish, or preparation of the report. Financial assistance through fellowship 
by the Council of Scientific and Industrial Research (CSIR) is gratefully acknowledged. Figures created with BioRender.com.

\section{Conflict of interest}

The authors declare no conflict of interest

Tables

Table 1. Identification of $L$. donovani infection in domestic animals through molecular and serological methods.

\begin{tabular}{llll}
\hline Country & Location & Detection Method & Potential Reservoir Sp \\
\hline India & Bihar & PCR, rk39 ICT & Goat, Rat, Cow \\
& Thiruvananthapuram & PCR & Dog \\
& Himachal Pradesh & rk 39 ICT & Dog \\
Nepal & Dharan & PCR & Buffalo, Cow, Goat \\
& Kathmandu & qPCR & Dog \\
Bangladesh & Mymensingh & rk39 ICT, PCR, qPCR & Dog \\
& Mymensingh & DAT, ELISA, rk39 ICT & Domestic Cattle, Goat \\
Sri Lanka & Matale & rk39 ICT, Microscopy & Dog \\
Ethiopia & Alduba, Dimeka, Kafta Humera & qPCR, PCR, DAT, NNN culture & Rodents \\
& Addis Zemen, Kafta Humera, Sheraro & PCR, ELISA, DAT, rk 39 ICT & Dog, Cow, Goat, Sheep \\
Sudan & Bandigueo, Urn Salala & DAT, ELISA & Donkey, Cow, Goat, Rat \\
& Blue Nile and Gedaref State & PCR, DAT & Dog \\
& Barbar El Fugara & NNN culture, IFAT & Dog \\
\hline
\end{tabular}

Abbreviations: $\mathrm{rK} 39=$ recombinant Leishmania K39 protein, $\mathrm{PCR}=$ Polymerase chain reaction; $\mathrm{ICT}=$ Immunochromatographic test; $\mathrm{qPCR}=$ quantitative polymerase chain reaction; $\mathrm{DAT}=$ Direct agglutination test; ELISA = Enzyme-linked immunosorbent assay; NNN= Novy-MacNeal-Nicolle medium; IFAT = Immunofluorescence antibody test

\section{Figure Legends}

Figure 1. Life cycles of zoonotic vs anthroponotic spread of Leishmania spp . Animals depicted have been documented as a blood source in Leishmania positive sand flies.

Figure 2. Distribution of $L$. donovani complex spp. and different transmission ecologies. AVL= anthroponotic VL, ZVL= zoonotic VL.

Figure 3. The skin as a predominant source forLeishmania parasites in different clinical settings. Sand flies use their serrated proboscis to probe the skin for dermal blood vessels creating a pool of blood from which to feed. Asymptomatic L. donovani infection provides a setting with productive immunity and a relatively low parasite load in the skin and blood. During symptomatic VL, high parasitemia and patches of highly parasitized macrophages in the skin lead to a highly increased chance of sand fly infection during feeding. PKDL is associated with an immune response againstLeishmania parasites in the skin and therefore is characterized by a significant parasite burden within dermal macrophages. After treatment for VL, parasitemia may diminish before parasites in the skin are eradicated. However, patients with both forms of PKDL have been found to be readily infectious to sand fly vectors. Nodular/papular PKDL patients, shown to have significantly increased parasite burden in the skin compared to macular PKDL, are more infectious to sand flies than macular PKDL patients.

\section{References}

Akhavan, A. A., H. Mirhendi, A. Khamesipour, M. H. Alimohammadian, Y. Rassi, P. Bates, S. Kamhawi, 
J. G. Valenzuela, M. H. Arandian and H. Abdoli, 2010: Leishmania species: detection and identification by nested PCR assay from skin samples of rodent reservoirs. Experimental parasitology, 126, 552-556.

Akhoundi, M., K. Kuhls, A. Cannet, J. Votýpka, P. Marty, P. Delaunay and D. Sereno, 2016: A historical overview of the classification, evolution, and dispersion of Leishmania parasites and sandflies. PLoS neglected tropical diseases, 10.

Akter, S., M. Z. Alam, R. Nakao, M. G. Yasin, H. Kato and K. Katakura, 2016: Molecular and serological evidence of Leishmania infection in stray dogs from visceral leishmaniasis-endemic areas of Bangladesh.The American journal of tropical medicine and hygiene, 95, 795-799.

Alam, M. S., D. Ghosh, M. G. M. Khan, M. F. Islam, D. Mondal, M. Itoh, M. N. Islam and R. Haque, 2011: Survey of domestic cattle for anti-Leishmania antibodies and LeishmaniaDNA in a visceral leishmaniasis endemic area of Bangladesh. BMC veterinary research, 7, 27.

Alam, M. Z., M. M. Rahman, S. Akter, M. H. Talukder and A. R. Dey, 2018: An investigation about the possible role of cattle and goats as reservoir hosts for Leishmania donovani in Bangladesh. Journal of vector borne diseases, 55, 242.

Alam, M. Z., M. G. Yasin, H. Kato, T. Sakurai and K. Katakura, 2013: PCR-based detection of Leishmania donovani DNA in a stray dog from a visceral leishmaniasis endemic focus in Bangladesh. Journal of Veterinary Medical Science, 75, 75-78.

Alvar, J., P. Aparicio, A. Aseffa, M. Den Boer, C. Canavate, J.-P. Dedet, L. Gradoni, R. Ter Horst, R. LópezVélez and J. Moreno, 2008: The relationship between leishmaniasis and AIDS: the second 10 years. Clinical microbiology reviews, 21, 334-359.

Baneth, G., A. F. Koutinas, L. Solano-Gallego, P. Bourdeau and L. Ferrer, 2008: Canine leishmaniosis-new concepts and insights on an expanding zoonosis: part one. Trends in parasitology, 24, 324-330.

Barnett, P. G., S. Singh, C. Bern, A. W. Hightower and S. Sundar, 2005: Virgin soil: the spread of visceral leishmaniasis into Uttar Pradesh, India. The American journal of tropical medicine and hygiene, 73, 720-725.

Bern, C., O. Courtenay and J. Alvar, 2010: Of cattle, sand flies and men: a systematic review of risk factor analyses for South Asian visceral leishmaniasis and implications for elimination. PLoS neglected tropical diseases, 4.

Bern, C., A. W. Hightower, R. Chowdhury, M. Ali, J. Amann, Y. Wagatsuma, R. Haque, K. Kurkjian, L. E. Vaz and M. Begum, 2005: Risk factors for kala-azar in Bangladesh. Emerging infectious diseases, 11, 655.

Bhattarai, N. R., G. Van der Auwera, S. Rijal, A. Picado, N. Speybroeck, B. Khanal, S. De Doncker, M. L. Das, B. Ostyn and C. Davies, 2010: Domestic animals and epidemiology of visceral leishmaniasis, Nepal.Emerging infectious diseases, 16, 231.

Blackwell, J., M. Fakiola, M. Ibrahim, S. Jamieson, S. Jeronimo, E. Miller, A. Mishra, H. Mohamed, C. Peacock and M. Raju, 2009: Genetics and visceral leishmaniasis: of mice and man. Parasite immunology,31, 254-266.

Burza, S., S. L. Croft and M. Boelaert, 2018: Leishmaniasis. The Lancet, 392, 951-970.

Callaway, E., 2016: Dogs thwart effort to eradicate Guinea worm.Nature News, 529, 10.

Cameron, M. M., A. Acosta-Serrano, C. Bern, M. Boelaert, M. Den Boer, S. Burza, L. A. Chapman, A. Chaskopoulou, M. Coleman and O. Courtenay, 2016: Understanding the transmission dynamics of Leishmania donovani to provide robust evidence for interventions to eliminate visceral leishmaniasis in Bihar, India. Parasites 85 vectors, $9,25$.

Coffeng, L. E., E. A. Le Rutte, J. Muñoz, E. R. Adams, J. M. Prada, S. J. de Vlas and G. F. Medley, 2019: Impact of Changes in Detection Effort on Control of Visceral Leishmaniasis in the Indian Subcontinent. The 
Journal of Infectious Diseases, 221, S546-S553.

Courtenay, O., D. Macdonald, R. Lainson, J. Shaw and C. Dyes, 1994: Epidemiology of canine leishmaniasis: a comparative serological study of dogs and foxes in Amazon Brazil. Parasitology, 109, 273-279.

Courtenay, O., N. C. Peters, M. E. Rogers and C. Bern, 2017: Combining epidemiology with basic biology of sand flies, parasites, and hosts to inform leishmaniasis transmission dynamics and control. PLoS pathogens, 13.

Courtenay, O., R. Quinnell, L. Garcez and C. Dye, 2002a: Low infectiousness of a wildlife host of Leishmania infantum: the crab-eating fox is not important for transmission. Parasitology,125, 407-414.

Courtenay, O., R. J. Quinnell, L. M. Garcez, J. J. Shaw and C. Dye, 2002b: Infectiousness in a cohort of Brazilian dogs: why culling fails to control visceral leishmaniasis in areas of high transmission. The Journal of infectious diseases, 186, 1314-1320.

Custodio, E., E. Gadisa, L. Sordo, I. Cruz, J. Moreno, J. Nieto, C. Chicharro, A. Aseffa, Z. Abraham and T. Hailu, 2012: Factors associated with Leishmania asymptomatic infection: results from a cross-sectional survey in highland northern Ethiopia. PLoS neglected tropical diseases, 6.

Das, V. N. R., R. N. Pandey, N. A. Siddiqui, L. A. Chapman, V. Kumar, K. Pandey, G. Matlashewski and P. Das, 2016: Longitudinal study of transmission in households with visceral leishmaniasis, asymptomatic infections and PKDL in highly endemic villages in Bihar, India.PLoS neglected tropical diseases, 10.

Daszak, P., A. A. Cunningham and A. D. Hyatt, 2001: Anthropogenic environmental change and the emergence of infectious diseases in wildlife. Acta tropica, 78, 103-116.

Dereure, J., M. Boni, F. Pratlong, M. E. H. Osman, B. Bucheton, S. El-Safi, E. Feugier, M. Musa, B. Davoust and A. Dessein, 2000: Visceral leishmaniasis in Sudan: first identifications of Leishmania from dogs. Transactions of the Royal Society of Tropical Medicine and Hygiene, 94, 154-155.

Dereure, J., S. H. El-Safi, B. Bucheton, M. Boni, M. M. Kheir, B. Davoust, F. Pratlong, E. Feugier, M. Lambert and A. Dessein, 2003: Visceral leishmaniasis in eastern Sudan: parasite identification in humans and dogs; host-parasite relationships. Microbes and infection, 5, 1103-1108.

Desjeux, P., 2004: Leishmaniasis: current situation and new perspectives. Comparative immunology, microbiology and infectious diseases, 27, 305-318.

Díaz-Regañón, D., B. Agulla, B. Piya, N. Fernández-Ruiz, A. Villaescusa, M. García-Sancho, F. RodríguezFranco and Á. Sainz, 2020: Stray dogs in Nepal have high prevalence of vector-borne pathogens: a molecular survey. Parasites 83 Vectors, 13, 174.

Eberhard, M. L., E. Ruiz-Tiben, D. R. Hopkins, C. Farrell, F. Toe, A. Weiss, P. C. Withers, M. H. Jenks, E. A. Thiele, J. A. Cotton, Z. Hance, N. Holroyd, V. A. Cama, M. A. Tahir and T. Mounda, 2014: The peculiar epidemiology of dracunculiasis in Chad. Am J Trop Med Hyg, 90, 61-70.

Esch, K. J. and C. A. Petersen, 2013: Transmission and epidemiology of zoonotic protozoal diseases of companion animals. Clinical microbiology reviews, 26, 58-85.

Esch, K. J., N. N. Pontes, P. Arruda, A. O'Connor, L. Morais, S. M. Jeronimo and C. A. Petersen, 2012: Preventing zoonotic canine leishmaniasis in northeastern Brazil: pet attachment and adoption of community leishmania prevention. The American journal of tropical medicine and hygiene, 87, 822-831.

Espinosa, O. A., M. G. Serrano, E. P. Camargo, M. M. G. Teixeira and J. J. Shaw, 2018: An appraisal of the taxonomy and nomenclature of trypanosomatids presently classified as Leishmania and Endotrypanum.Parasitology, 145, 430-442.

Esteva, L., C. Vargas and C. V. de León, 2017: The role of asymptomatics and dogs on leishmaniasis propagation. Mathematical biosciences,293, 46-55. 
Fischer, D., P. Moeller, S. M. Thomas, T. J. Naucke and C. Beierkuhnlein, 2011: Combining climatic projections and dispersal ability: a method for estimating the responses of sandfly vector species to climate change. PLoS neglected tropical diseases, 5 .

Franssen, S. U., C. Durrant, O. Stark, B. Moser, T. Downing, H. Imamura, J.-C. Dujardin, M. J. Sanders, I. Mauricio and M. A. J. E. Miles, 2020: Global genome diversity of the Leishmania donovani complex. 9, e51243.

Galán-Puchades, M. T., 2017: WHO delays guinea-worm disease eradication to 2020: are dogs the sole culprits? The Lancet Infectious Diseases, 17, 1124-1125.

Gavgani, A. M., M. Hodjati, H. Mohite and C. Davies, 2002: Effect of insecticide-impregnated dog collars on incidence of zoonotic visceral leishmaniasis in Iranian children: a matchedcluster randomised trial. The Lancet, 360, 374-379.

Gidwani, K., A. Picado, B. Ostyn, S. P. Singh, R. Kumar, B. Khanal, V. Lejon, F. Chappuis, M. Boelaert and S. Sundar, 2011: Persistence of Leishmania donovani antibodies in past visceral leishmaniasis cases in India. Clin. Vaccine Immunol., 18, 346-348.

Hamarsheh, O., A. Nasereddin, S. Damaj, S. Sawalha, H. Al-Jawabreh, K. Azmi, A. Amro, S. Ereqat, Z. Abdeen and A. Al-Jawabreh, 2012: Serological and molecular survey of Leishmania parasites in apparently healthy dogs in the West Bank, Palestine. Parasites $\&$ vectors, $5,183$.

Hassan, M. a. M., O. F. Osman, F. M. A. El-Raba'a, H. D. F. H. Schallig and D.-E. A. Elnaiem, 2009: Role of the domestic dog as a reservoir host of Leishmania donovani in eastern Sudan. Parasites 85 Vectors, 2, 26.

Haydon, D. T., S. Cleaveland, L. H. Taylor and M. K. Laurenson, 2002: Identifying reservoirs of infection: a conceptual and practical challenge. Emerging infectious diseases, 8, 1468-1473.

Huda, M. M., S. Hirve, N. A. Siddiqui, P. Malaviya, M. R. Banjara, P. Das, S. Kansal, C. K. Gurung, E. Naznin and S. J. B. p. h. Rijal, 2012: Active case detection in national visceral leishmaniasis elimination programs in Bangladesh, India, and Nepal: feasibility, performance and costs. 12, 1001.

Jambulingam, P., N. P. Kumar, S. Nandakumar, K. Paily and R. Srinivasan, 2017: Domestic dogs as reservoir hosts for Leishmania donovani in the southernmost Western Ghats in India. Acta tropica, 171, 64-67.

Kalayou, S., H. Tadelle, A. Bsrat, N. Abebe, M. Haileselassie and H. Schallig, 2011: Serological Evidence of Leishmania donovani Infection in Apparently Healthy Dogs using Direct Agglutination Test (DAT) and rk39 Dipstick Tests in Kafta Humera, north-west Ethiopia. Transboundary and emerging diseases, 58, 255-262.

Kamhawi, S. and T. D. Serafim, 2017: Patchy parasitized skin governs Leishmania donovani transmission to sand flies. Trends in parasitology, 33, 748-750.

Kassahun, A., J. Sadlova, V. Dvorak, T. Kostalova, I. Rohousova, D. Frynta, T. Aghova, D. Yasur-Landau, W. Lemma and A. Hailu, 2015: Detection of Leishmania donovani and L. tropica in Ethiopian wild rodents. Acta tropica, 145, 39-44.

Keeling, M., 1999: Spatial models of interacting populations. pp. 64-99. Wiley Online Library.

Le Rutte, E. A., L. E. Coffeng, D. M. Bontje, E. C. Hasker, J. A. R. Postigo, D. Argaw, M. C. Boelaert and S. J. De Vlas, 2016: Feasibility of eliminating visceral leishmaniasis from the Indian subcontinent: explorations with a set of deterministic age-structured transmission models. Parasites $\&$ vectors, 9, 24.

Lemma, W., A. Bizuneh, H. Tekie, H. Belay, H. Wondimu, A. Kassahun, W. Shiferaw, M. Balkew, I. Abassi and G. Baneth, 2017: Preliminary study on investigation of zoonotic visceral leishmaniasis in endemic foci of Ethiopia by detecting Leishmania infections in rodents. Asian Pacific journal of tropical medicine, 10, 418-422. 
Lukeš, J., I. L. Mauricio, G. Schönian, J.-C. Dujardin, K. Soteriadou, J.-P. Dedet, K. Kuhls, K. W. Q. Tintaya, M. Jirků and E. Chocholová, 2007: Evolutionary and geographical history of the Leishmania donovani complex with a revision of current taxonomy. Proceedings of the National Academy of Sciences, 104, 9375-9380.

Malaviya, P., A. Picado, S. P. Singh, E. Hasker, R. P. Singh, M. Boelaert and S. Sundar, 2011: Visceral leishmaniasis in Muzaffarpur district, Bihar, India from 1990 to 2008. PloS one, 6.

Marcili, A., M. A. Speranca, A. P. da Costa, F. Madeira Mde, H. S. Soares, O. Sanches Cde, C. Acosta Ida, A. Girotto, A. H. Minervino, M. C. Horta, J. J. Shaw and S. M. Gennari, 2014: Phylogenetic relationships of Leishmania species based on trypanosomatid barcode (SSU rDNA) and gGAPDH genes: Taxonomic revision of Leishmania (L.) infantum chagasi in South America. Infect Genet Evol, 25, 44-51.

McMahon-Pratt, D. and J. Alexander, 2004: Does the Leishmania major paradigm of pathogenesis and protection hold for New World cutaneous leishmaniases or the visceral disease? Immunological reviews,201, 206-224.

Molina, R., D. Ghosh, E. Carrillo, S. Monnerat, C. Bern, D. Mondal and J. Alvar, 2017: Infectivity of post-kala-azar dermal leishmaniasis patients to sand flies: revisiting a proof of concept in the context of the kala-azar elimination program in the Indian subcontinent. Clinical Infectious Diseases, 65, 150-153.

Molina, R., M. Jimenez, I. Cruz, A. Iriso, I. Martin-Martin, O. Sevillano, S. Melero and J. Bernal, 2012: The hare (Lepus granatensis) as potential sylvatic reservoir of Leishmania infantum in Spain. Veterinary parasitology, 190, 268-271.

Molina, R., M. Jimenez, J. Garcia-Martinez, J. V. San Martin, E. Carrillo, C. Sanchez, J. Moreno, F. Alves and J. J. P. N. T. D. Alvar, 2020: Role of asymptomatic and symptomatic humans as reservoirs of visceral leishmaniasis in a Mediterranean context. 14, e0008253.

Mondal, D., C. Bern, D. Ghosh, M. Rashid, R. Molina, R. Chowdhury, R. Nath, P. Ghosh, L. A. Chapman and A. Alim, 2019: Quantifying the Infectiousness of Post-Kala-Azar Dermal Leishmaniasis Toward Sand Flies. Clinical Infectious Diseases, 69, 251-258.

Mondal, D., S. P. Singh, N. Kumar, A. Joshi, S. Sundar, P. Das, H. Siddhivinayak, A. Kroeger and M. J. P. N. T. D. Boelaert, 2009: Visceral leishmaniasis elimination programme in India, Bangladesh, and Nepal: reshaping the case finding/case management strategy. 3, e355.

Monge-Maillo, B. and R. Lopez-Velez, 2013: Therapeutic Options for Old World Cutaneous Leishmaniasis and New World Cutaneous and Mucocutaneous Leishmaniasis. Drugs, 73, 1889-1920.

Mukhopadhyay, D., J. E. Dalton, P. M. Kaye and M. Chatterjee, 2014: Post kala-azar dermal leishmaniasis: an unresolved mystery. Trends in parasitology, 30, 65-74.

Mukhtar, M. M., A. Sharief, S. El Saffi, A. Harith, T. Higazzi, A. Adam and H. S. Abdalla, 2000: Detection of antibodies to Leishmania donovani in animals in a kala-azar endemic region in eastern Sudan: a preliminary report. Transactions of the Royal Society of Tropical Medicine and Hygiene, 94, 33-36.

Nasereddin, A., G. Baneth, G. Schonian, M. Kanaan and C. L. Jaffe, 2005: Molecular fingerprinting of Leishmania infantum strains following an outbreak of visceral leishmaniasis in central Israel. Journal of clinical microbiology, 43, 6054-6059.

Nawaratna, S. S., D. J. Weilgama and K. Rajapaksha, 2009: Cutaneous leishmaniasis in Sri Lanka: a study of possible animal reservoirs.International Journal of Infectious Diseases, 13, 513-517.

Oryan, A. and M. Akbari, 2016: Worldwide risk factors in leishmaniasis.Asian Pacific Journal of Tropical Medicine, 9, 925-932.

Ostyn, B., K. Gidwani, B. Khanal, A. Picado, F. Chappuis, S. P. Singh, S. Rijal, S. Sundar and M. Boelaert, 2011: Incidence of symptomatic and asymptomatic Leishmania donovani infections in high-endemic foci in 
India and Nepal: a prospective study. PLoS neglected tropical diseases, 5.

Palatnik-de-Sousa, C. B. and M. J. Day, 2011: One Health: the global challenge of epidemic and endemic leishmaniasis. Parasites \& vectors, 4, 197.

Patz, J. A., T. K. Graczyk, N. Geller and A. Y. Vittor, 2000: Effects of environmental change on emerging parasitic diseases. International journal for parasitology, 30, 1395-1405.

Perry, D., K. Dixon, R. Garlapati, A. Gendernalik, D. Poche and R. Poche, 2013: Visceral leishmaniasis prevalence and associated risk factors in the saran district of Bihar, India, from 2009 to July of 2011. The American journal of tropical medicine and hygiene, 88, 778-784.

Picado, A., A. P. Dash, S. Bhattacharya and M. Boelaert, 2012: Vector control interventions for visceral leishmaniasis elimination initiative in South Asia, 2005-2010. The Indian journal of medical research,136, 22 .

Poche, D. M., R. B. Garlapati, S. Mukherjee, Z. Torres-Poche, E. Hasker, T. Rahman, A. Bharti, V. P. Tripathi, S. Prakash and R. Chaubey, 2018: Bionomics of Phlebotomus argentipes in villages in Bihar, India with insights into efficacy of IRS-based control measures. PLoS neglected tropical diseases, 12.

Programme, D. o. N. V. B. D. C., 2017: Accelerated Plan for Kala-azar Elimination 2017. Ministry of Health and Family Welfare, government of India, New Delhi .

Quinnell, R. J. and O. Courtenay, 2009: Transmission, reservoir hosts and control of zoonotic visceral leishmaniasis. Parasitology,136, 1915-1934.

Ranjan, A., D. Sur, V. P. Singh, N. A. Siddique, B. Manna, C. S. Lal, P. K. Sinha, K. Kishore and S. K. Bhattacharya, 2005: Risk factors for Indian kala-azar. The American journal of tropical medicine and hygiene, $73,74-78$.

Raymond, R. W., C. P. McHugh, L. R. Witt and S. F. Kerr, 2003: Temporal and spatial distribution of Leishmania mexicana infections in a population of Neotoma micropus. Memorias do Instituto Oswaldo Cruz, 98, 171-180.

Ready, P., 2008: Leishmaniasis emergence and climate change. Rev Sci Tech, 27, 399-412.

Ready, P. D., 2013: Biology of phlebotomine sand flies as vectors of disease agents. Annual review of entomology, 58.

Reithinger, R., J.-C. Dujardin, H. Louzir, C. Pirmez, B. Alexander and S. Brooker, 2007: Cutaneous leishmaniasis. The Lancet infectious diseases, 7, 581-596.

Rijal, S., S. Sundar, D. Mondal, P. Das, J. Alvar and M. J. b. Boelaert, 2019: Eliminating visceral leishmaniasis in South Asia: the road ahead. 364.

Rohousova, I., D. Talmi-Frank, T. Kostalova, N. Polanska, T. Lestinova, A. Kassahun, D. Yasur-Landau, C. Maia, R. King and J. Votypka, 2015: Exposure to Leishmania spp. and sand flies in domestic animals in northwestern Ethiopia. Parasites 83 vectors, 8, 360.

Rosypal, A., S. Tripp, C. Kinlaw, S. Hailemariam, R. Tidwell, D. Lindsay, R. Rajapakse, C. Sreekumar and J. Dubey, 2010: Surveillance for antibodies to Leishmania spp. in dogs from Sri Lanka. Journal of Parasitology, 96, 230-231.

Ruiz-Fons, F., E. Ferroglio and C. Gortazar, 2013: Leishmania infantum in free-ranging hares, Spain, 20042010. Eurosurveillance, 18, 20541.

Shamboul, K. M., A. M. El Bagir, M. O. El Sayed, S. A. Saeed, H. Abdalla and O. Omran, 2009: Identification of Leishmania donovani from infected dogs at a dormant focus of VL in Blue Nile State, Sudan. J Genet Eng Biotechnol, 7, 27-31. 
Sharma, N. L., V. K. Mahajan, A. K. Negi and G. K. Verma, 2009: The rK39 immunochromatic dipstick testing: a study for K39 seroprevalence in dogs and human leishmaniasis patients for possible animal reservoir of cutaneous and visceral leishmaniasis in endemic focus of Satluj river valley of Himachal Pradesh (India). Indian Journal of Dermatology, Venereology, and Leprology, 75, 52.

Shaw, J. J., 2006: Further thoughts on the use of the name Leishmania (Leishmania) infantum chagasi for the aetiological agent of American visceral leishmaniasis. Mem Inst Oswaldo Cruz, 101, 577-579.

Singh, N., J. Mishra, R. Singh and S. Singh, 2013: Animal reservoirs of visceral leishmaniasis in India. Journal of Parasitology, 99, 64-67.

Singh, O. P., E. Hasker, M. Boelaert, D. Sacks and S. J. P. N. T. D. Sundar, 2020a: Xenodiagnosis to address key questions in visceral leishmaniasis control and elimination. 14, e0008363.

Singh, O. P., E. Hasker, D. Sacks, M. Boelaert and S. Sundar, 2014: Asymptomatic Leishmania infection: a new challenge for Leishmania control. Clinical Infectious Diseases, 58, 1424-1429.

Singh, O. P., P. Tiwary, A. K. Kushwaha, S. K. Singh, D. K. Singh and S. Sundar, 2020b: Xenodiagnosis to evaluate the infectiousness of humans to sand flies in an area endemic for visceral leishmaniasis in Bihar, India: a transmission dynamics study. The Lancet Microbe, 2/1.

Singh, S., E. Hasker, A. Picado, K. Gidwani, P. Malaviya, R. Singh, M. Boelaert and S. Sundar, 2010: Risk factors for visceral leishmaniasis in India: further evidence on the role of domestic animals. Tropical Medicine $\mathscr{E}$ International Health, 15, 29-35.

Stauch, A., R. R. Sarkar, A. Picado, B. Ostyn, S. Sundar, S. Rijal, M. Boelaert, J.-C. Dujardin and H.-P. Duerr, 2011: Visceral leishmaniasis in the Indian subcontinent: modelling epidemiology and control.PLoS neglected tropical diseases, 5 .

Thakur, C. P., 2000: Socio-economics of visceral leishmaniasis in Bihar (India). Trans R Soc Trop Med Hyg, 94, 156-157.

Thiele, E. A., M. L. Eberhard, J. A. Cotton, C. Durrant, J. Berg, K. Hamm and E. Ruiz-Tiben, 2018: Population genetic analysis of Chadian Guinea worms reveals that human and non-human hosts share common parasite populations. PLoS Negl Trop Dis, 12, e0006747.

Tiwary, P., S. K. Singh, A. K. Kushwaha, E. Rowton, D. Sacks, O. P. Singh, S. Sundar and P. Lawyer, 2017: Establishing, expanding, and certifying a closed colony of Phlebotomus argentipes (Diptera: Psychodidae) for xenodiagnostic studies at the Kala Azar Medical Research Center, Muzaffarpur, Bihar, India. Journal of medical entomology, 54, 1129-1139.

WHO, 2005: Regional strategic framework for elimination of kala-azar from the South-East Asia region (2005-2015). New Delhi (India).

WHO, 2020: Global leishmaniasis surveillance, 2017-2018, and first report on 5 additional indicatorsSurveillance mondiale de la leishmaniose, 2017-2018, et premier rapport sur 5 indicateurs supplementaires. 95, 265-279.

Zijlstra, E. E., F. Alves, S. Rijal, B. Arana and J. Alvar, 2017: Post-kala-azar dermal leishmaniasis in the Indian subcontinent: A threat to the South-East Asia Region Kala-azar Elimination Programme.PLoS neglected tropical diseases, 11. 

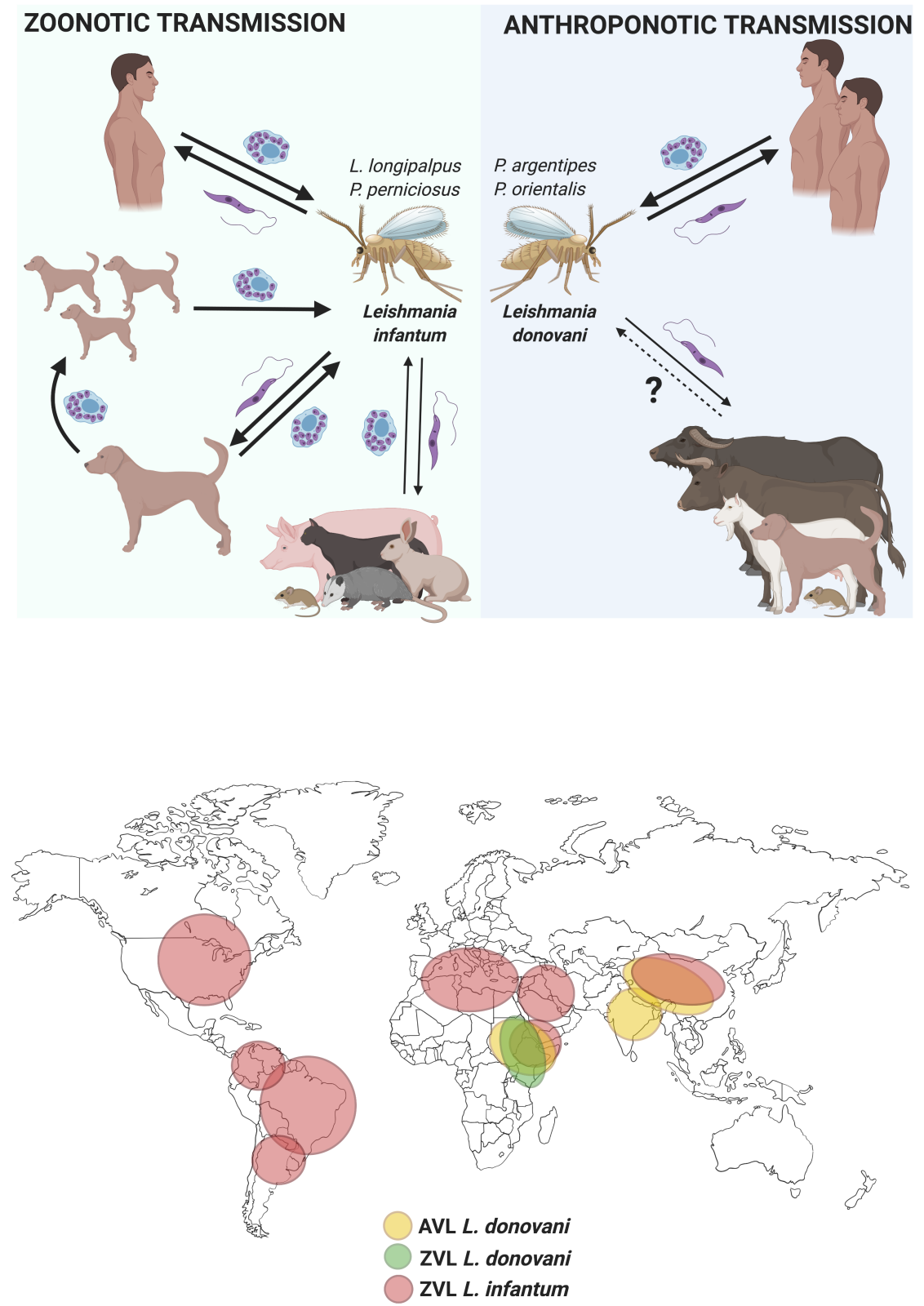
ASYMPTOMATIC INFECTION

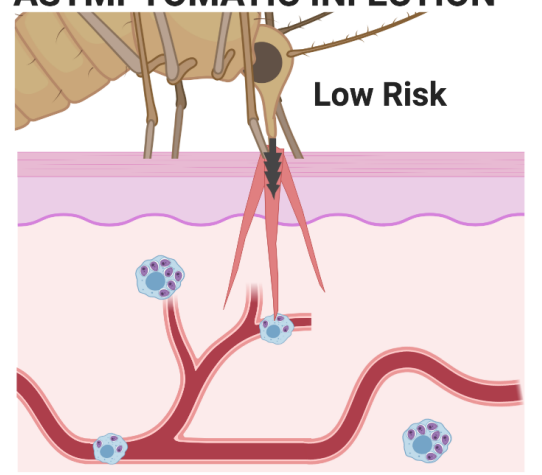

NODULAR/PAPULAR PKDL

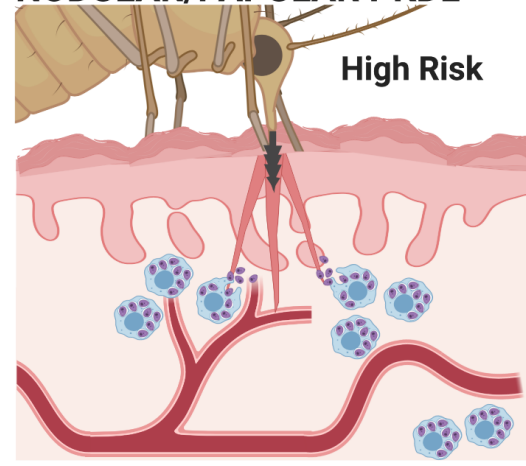

VISCERAL LEISHMANIASIS

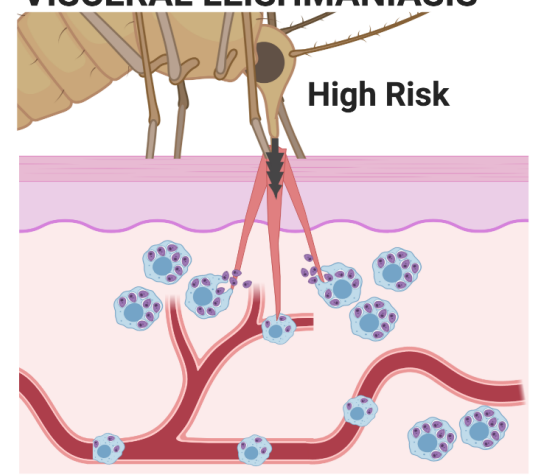

MACULAR PKDL

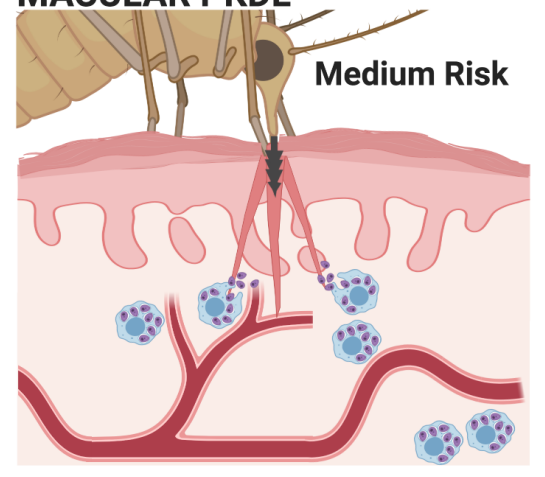

